\title{
Effect of location of delamination on free vibration of cross-ply conical shells
}

\author{
Sudip Dey* and Amit Karmakar \\ Mechanical Engineering Department, Jadavpur University, Kolkata, India
}

Received 29 July 2011

Revised 17 October 2011

\begin{abstract}
Location of delamination is a triggering parameter for structural instability of laminated composites. In this paper, a finite element method is employed to determine the effects of location of delamination on free vibration characteristics of graphite-epoxy cross-ply composite pre-twisted shallow conical shells. The generalized dynamic equilibrium equation is derived from Lagrange's equation of motion neglecting Coriolis effect for moderate rotational speeds. The formulation is exercised by using an eight noded isoparametric plate bending element based on Mindlin's theory. Multi-point constraint algorithm is utilized to ensure the compatibility of deformation and equilibrium of resultant forces and moments at the delamination crack front. The standard eigen value problem is solved by applying the QR iteration algorithm. Finite element codes are developed to obtain the numerical results concerning the effects of location of delamination, twist angle and rotational speed on the natural frequencies of cross-ply composite shallow conical shells. The mode shapes are also depicted for a typical laminate configuration. Numerical results obtained from parametric studies of both symmetric and anti-symmetric cross-ply laminates are the first known non-dimensional natural frequencies for the type of analyses carried out here.
\end{abstract}

Keywords: Delamination, finite element, cross-ply, conical shell, multi-point constraint, mode shape, vibration

\section{Nomenclature}

$\begin{array}{ll}\mathrm{R}_{x} & \text { Radius of curvature in x-direction } \\ \mathrm{R}_{y} & \text { Radius of curvature in y-direction } \\ \mathrm{R}_{x y} & \text { Radius of twist } \\ \Psi & \text { Twist angle } \\ \Omega & \text { Non-dimensional speed of rotation }\left(=\Omega^{\prime} / \omega_{o}\right) \\ \Omega^{\prime} & \text { Actual angular speed of rotation } \\ \omega_{o} & \text { Fundamental natural frequency of a non-rotating shell } \\ \rho & \text { Mass density } \\ \mathrm{L} & \text { Length } \\ b_{o} & \text { Reference width } \\ \nu & \text { Poisson's ratio } \\ \mathrm{h} & \text { Thickness } \\ \mathrm{d} & \text { Distance of centerline of delamination from clamped (fixed) end } \\ \mathrm{a} & \text { Crack length } \\ \theta_{v} & \text { Vertex angle } \\ \theta_{o} & \text { Base subtended angle of cone } \\ \mathrm{E}_{1}, \mathrm{E}_{2} & \text { Elastic moduli along 1 and 2 axes } \\ \mathrm{G}_{12}, \mathrm{G}_{13}, \mathrm{G}_{23} & \text { Shear moduli along 1-2, 1-3 and 2-3 planes } \\ & \end{array}$

${ }^{*}$ Corresponding author: Tel.: +9133 24847230; E-mail: infosudip@gmail.com. 


$\begin{array}{ll}{[\mathrm{M}]} & \text { Global mass matrix } \\ {[\mathrm{K}]} & \text { Elastic stiffness matrix } \\ {\left[\mathrm{K}_{\sigma}\right]} & \text { Geometric stiffness matrix } \\ \{\delta\} & \text { Global displacement vector } \\ \lambda & \text { Non-dimensional frequency } \\ \mathrm{u}_{j}, \mathrm{v}_{j}, \mathrm{w}_{j}, & \text { Nodal displacements } \\ \mathrm{u}^{\prime}, \mathrm{v}^{\prime}{ }_{j}, \mathrm{w}^{\prime}{ }_{j} & \text { Mid-plane displacements } \\ \theta_{x}, \theta_{y} & \text { Rotation about } x \text { and } y \text { axes } \\ \{\mathrm{Q}\} & \text { Transverse shear resultants } \\ {[\mathrm{A}]} & \text { Extension coefficient } \\ {[\mathrm{B}]} & \text { Bending-extension coupling coefficient } \\ {[\mathrm{D}]} & \text { Bending stiffness coefficients } \\ \{\mathrm{k}\} & \text { Curvature vector } \\ \{\mathrm{N}\} & \text { In-plane stress-resultants } \\ \{\mathrm{M}\} & \text { Moment resultants } \\ \{\varepsilon\} & \text { Strains vector } \\ \eta, \xi & \text { Local natural coordinates of the element } \\ \mathrm{X}, \mathrm{y}, \mathrm{z} & \text { Local coordinate axes (plate coordinate system) } \\ \mathrm{n} & \text { Number of layers } \\ \mathrm{L} / \mathrm{s} & \text { Aspect ratio } \\ \omega_{n} & \text { Natural frequency of rotating shell } \\ \mathrm{ND} & \text { No delamination or undelaminated cases } \\ \mathrm{NDFF} & \text { Non-dimensional fundamental natural frequency } \\ \mathrm{NDSF} & \text { Non-dimensional second natural frequency } \\ & \end{array}$

\section{Introduction}

Extensive uses of composite laminated structures can be found in weight-sensitive applications such as turbomachinery engines. Rotating pretwisted conical shells with low aspect ratio can be idealized as turbo-machinery blades (Fig. 1). Composite material has the increasing demand due to its high specific strength and high specific stiffness with high compliance to design aspects as well as their cost-effectiveness. Delamination or inter-laminar debonding or separation of layers of constituting laminae is the most common feared mode of damage in the composite structure. The delaminated structure exhibits new vibration characteristics depending on size and location of delamination. A critical size and location of delamination can result in degradation of strength, structural instability and can lead to failure. The presence of invisible delamination can be detected with the help of prior knowledge of natural frequencies for composite laminates containing delamination. Therefore, designers must have a prior profound understanding of dynamic behaviour of composite structures to ensure the operational safety.

The first established work on pretwisted composite plates was carried out by Qatu and Leissa [15] to determine the natural frequencies of stationary plates employing laminated shallow shell theory using Ritz method. Liew et al. [11] investigated on pretwisted conical shell to find out the vibratory characteristics of stationary conical shell by using Ritz procedure and by using the same method, the first known three dimensional continuum vibration analysis including full geometric non-linearities and centrifugal accelerations in composite blades was carried out by McGee and Chu [19]. The work on delamination buckling and growth of flat cross-ply laminates was also investigated by Sallam and Simitses [24]. Regarding delamination model, two worth mentioning investigations were carried out. It included analytical and experimental determination of natural frequencies of delaminated composite beam by Shen and Grady [13] and the second one dealt with finite element treatment of the delaminated composite cantilever beam and plate by Krawczuk et al. [14] for free vibration analyses. Liang et al. [23] studied natural vibration of symmetric cross-ply laminated composite conical plate shell by using transfer matrix method, while Narita et al. [27] investigated the free vibration characteristics of cross-ply laminated cylindrical shell by leveraging finite element method. On the other hand, Takeda and Ogihara [17] experimentally investigated on delamination 


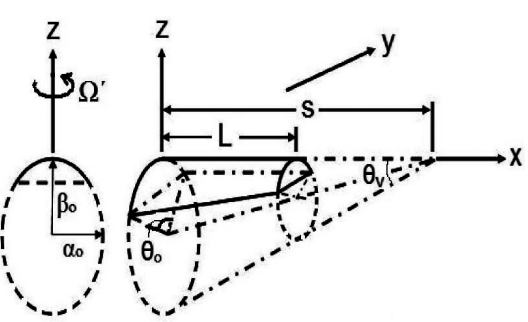

(a)
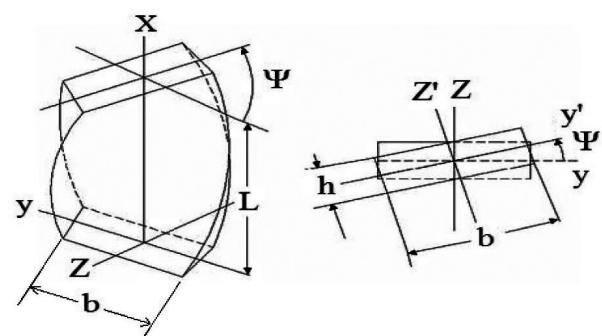

(b)

Fig. 1. Geometry of (a) Untwisted shallow conical shell and (b) Twisted plate.

initiation and growth from the tips of the transverse cracks in CFRP cross-ply laminates, while Todoroki et al. [3] experimentally monitored location and size of delamination crack of graphite-epoxy composite plate employing electric resistance change method. Later on, Berthelot and Le Corre [9] analyzed statistically the progression of transverse cracking delamination process induced in cross-ply laminates subjected to tensile loading. Toyama et al. [18] analytically investigated on the effects of transverse cracking and delamination on the mode velocity in GFRP and CFRP cross-ply laminates. Rebière and Gamby [8] employed variational approach to model the behaviour of composite cross-ply laminates damaged by transverse, longitudinal cracking and delamination. On the contrary, free vibration of laminated composite employing finite element method analyzed by both Aydogdu and Timarci [12] as well as Tripathi et al. [26]. Further, Lee et al. [7] exhibited the vibration analysis of twisted cantilevered conical composite shell by using finite element method based on the Hellinger-Reissner principle. Of late, Karmakar and kishimoto [1] investigated on free vibration of delaminated rotating cylindrical shells. Besides investigation on single delamination, significant work also incurred on multiple delaminations. Considering multiple delamination, Aymerich et al. [6] simulated in impacted cross-ply laminates based on cohesive interface elements by using finite element model. Parhi et al. [20] numerically investigated the failure analysis of multiple delaminated composite plate due to bending and impact.

Therefore, considering all the above research findings, it is clear that no major attention has been made to the initially stressed delaminated rotating composite plates or shells. It is also observed that only a limited number of investigations have been carried out related to laminated composite cantilever conical shells with initial twist and subsequently the research findings are very limited and scanty. To the best of the authors' knowledge, there is no literature available, which deals with delaminated pretwisted rotating composite cross-ply conical shells. To fill up this apparent void, the present analyses employed a finite-element based approach to investigate the effect of location of delamination on free vibration characteristics of graphite-epoxy pretwisted shallow cross-ply composite conical shells. The analyses are carried out using an eight-noded isoparametric plate bending element considering the effects of transverse shear deformation and rotary inertia based Mindlin's theory. The undelaminated region is modeled by a single layer of plate elements while the delaminated region is modeled using two layers of plate elements whose interface contains the delamination. To ensure the compatibility of deformation and equilibrium of resultant forces and moments at the delamination crack front a multi-point constraint algorithm [5] is incorporated which leads to anti-symmetric element stiffness matrices. The QR iteration algorithm [10] is utilized to solve the standard eigenvalue problem. The first two non-dimensional natural frequencies are obtained considering the effects of triggering parameters like the twist angle, location of delamination, rotational speed and fiber-orientation angle. This paper presents a finite element based numerical approach to determine the effect of location of delamination on non-dimensional natural frequencies of graphite-epoxy pretwisted shallow cross-ply composite conical shells neglecting effect of dynamic contact between delaminated layers.

\section{Mathematical formulation}

A shallow shell is characterized by its middle surface defined by the equation [4],

$$
z=-\frac{1}{2}\left[\frac{x^{2}}{R_{x}}+2 \frac{x y}{R_{x y}}+\frac{y^{2}}{R_{y}}\right]
$$


where $R_{x}, R_{y}$ and $R_{x y}$ denote the radii of curvature in the $x$ and $y$ directions and radius of twist, respectively. The radius of twist $\left(R_{x y}\right)$, length $(L)$ of the shell and twist angle $(\Psi)$ are related as,

$$
\tan \psi=-\frac{L}{R_{x y}}
$$

The dynamic equilibrium equation for moderate rotational speeds neglecting Coriolis effect is derived employing Lagrange's equation of motion and the equation in global form is expressed as [2]

$$
[M]\{\ddot{\delta}\}+\left([K]+\left[K_{\sigma}\right]\right)\{\delta\}=\left\{F\left(\Omega^{2}\right)\right\}
$$

where $[M],[K],\left[K_{\sigma}\right]$ are global mass, elastic stiffness and geometric stiffness matrices [21], respectively. $\left\{\mathrm{F}\left(\Omega^{2}\right)\right\}$ is the nodal equivalent centrifugal forces and $\{\delta\}$ is the global displacement vector. $\left[\mathrm{K}_{\sigma}\right]$ depends on the initial stress distribution and is obtained by the iterative procedure upon solving,

$$
\left([K]+\left[K_{\sigma}\right]\right)\{\delta\}=\left\{F\left(\Omega^{2}\right)\right\}
$$

The natural frequencies $(\omega)$ are determined from the standard eigen value problem [10] which is represented below and is solved by the QR iteration algorithm,

$$
[A]\{\delta\}=\lambda\{\delta\}
$$

where

$$
[A]=\left([K]+\left[K_{\sigma}\right]\right)^{-1}[M]
$$

and

$$
\lambda=1 / \omega_{n}^{2}
$$

\subsection{Multi-point constraints}

The cross-sectional view of a typical delamination crack tip is shown in Fig. 2 [5] where the nodes of three plate elements meet together to form a common node. The undelaminated region is modeled by plate element 1 of thickness $\mathrm{h}$, and the delaminated region is modeled by plate elements 2 and 3 whose interface contains the delamination $\left(h_{2}\right.$ and $h_{3}$ are the thicknesses of the elements 2 and 3 , respectively). The elements 1,2 and 3 are freely allowed to deform prior to imposition of the constraints conditions. The nodal displacements of elements 2 and 3 at the crack tip are expressed as [5]

$$
\begin{aligned}
& u_{j}=u_{j}^{\prime}-\left(z-z_{j}^{\prime}\right) \theta_{x j} \\
& v_{j}=v_{j}^{\prime}-\left(z-z_{j}^{\prime}\right) \theta_{y j} \\
& w_{j}=w_{j}^{\prime}(\text { where, } j=2,3)
\end{aligned}
$$

where $u_{j}^{\prime}, v_{j}^{\prime}$ and $w_{j}^{\prime}$ are the mid-plane displacements and $z_{j}^{\prime}$ is the z-coordinate of mid-plane of element $j$ and $\theta_{x}$, $\theta_{y}$ are the rotations about $x$ and $y$ axes, respectively. The above equation also holds good for element 1 and $z_{1}^{\prime}$ equal to zero. The transverse displacements and rotations at a common node have values expressed as,

$$
\begin{aligned}
& w_{1}=w_{2}=w_{3}=w \\
& \theta_{x 1}=\theta_{x 2}=\theta_{x 3}=\theta_{x} \\
& \theta_{y 1}=\theta_{y 2}=\theta_{y 3}=\theta_{y}
\end{aligned}
$$

In-plane displacements of all the three elements at a crack tip are equal and they are related as,

$$
\begin{aligned}
& u_{2}^{\prime}=u_{1}^{\prime}-z_{2}^{\prime} \theta_{x} \\
& v_{2}^{\prime}=v_{1}^{\prime}-z_{2}^{\prime} \theta_{y}
\end{aligned}
$$




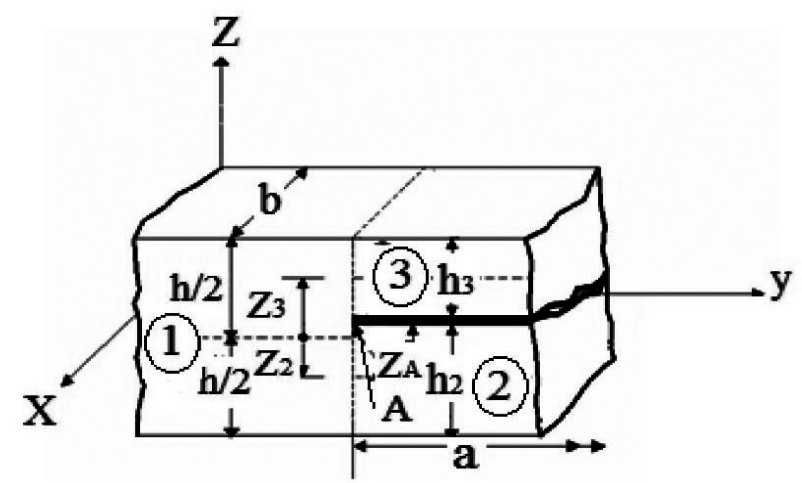

Fig. 2. Plate elements at a delamination crack tip [5].

$$
\begin{aligned}
& u_{3}^{\prime}=u_{1}^{\prime}-z_{3}^{\prime} \theta_{x} \\
& v_{3}^{\prime}=v_{1}^{\prime}-z_{3}^{\prime} \theta_{y}
\end{aligned}
$$

where $u_{1}^{\prime}$ is the mid-plane displacement of element 1. Equations (10) to (16) relating the nodal displacements and rotations of elements 1,2 and 3 at the delamination crack tip, are the multipoint constraint equations used in the finite element formulation to satisfy the compatibility of displacements and rotations. Mid-plane strains between elements 2 and 3 are related as [5],

$$
\left.\left\{\varepsilon^{\prime}\right\}_{j}=\left\{\varepsilon^{\prime}\right\}_{1}+z_{j}^{\prime}\{k\} \text { (where }=2,3\right)
$$

where $\left\{\varepsilon^{\prime}\right\}$ represents the strain vector and $\{k\}$ is the curvature vector being identical at the crack tip for elements 1,2 and 3 . This equation can be considered as a special case for element 1 when $z_{1}^{\prime}$ is equal to zero. In-plane stress-resultants, $\{\mathrm{N}\}$ and moment resultants, $\{\mathrm{M}\}$ of elements 2 and 3 can be expressed as [5],

$$
\begin{aligned}
& \{N\}_{j}=[A]_{j}\left\{\varepsilon^{\prime}\right\}_{1}+\left(z_{j}^{\prime}[A]_{j}+[B]_{j}\right)\{k\}(\text { where } j=2,3) \\
& \{M\}_{j}=[B]_{j}\left\{\varepsilon^{\prime}\right\}_{1}+\left(z_{j}^{\prime}[B]_{j}+[D]_{j}\right)\{k\}(\text { where } j=2,3) \\
& {[D]_{t}=\left[\begin{array}{ccc}
A_{i j} & z_{t}^{o} A_{i j}+B_{i j} & 0 \\
B_{i j} & z_{t}^{o} A_{i j}+D_{i j} & 0 \\
0 & 0 & S_{i j}
\end{array}\right]}
\end{aligned}
$$

where $[\mathrm{A}],[\mathrm{B}]$ and $[\mathrm{D}]$ are the extension, bending-extension coupling and bending stiffness coefficients of the composite laminate, respectively. The elasticity matrix for the $\mathrm{t}$-th sublaminate is then modified and is expressed in the form

where,

$$
\begin{aligned}
{\left[A_{i j}\right]_{t} } & =\int_{-h / 2+Z_{t}^{o}}^{h / 2+Z_{t}^{o}}[\bar{Q}] d z \\
{\left[B_{i j}\right]_{t}=} & \int_{-h / 2+Z_{t}^{o}}^{h / 2+Z_{t}^{o}}[\bar{Q}]\left(z-z_{t}^{o}\right) d z=\int_{-h / 2+Z_{t}^{o}}^{h / 2+Z_{t}^{o}}[\bar{Q}] z d z-z_{t}^{o}\left[A_{i j}\right]_{t} \\
{\left[D_{i j}\right]_{t}=} & \int_{-h / 2+Z_{t}^{o}}^{h / 2+Z_{t}^{o}}[\bar{Q}]\left(z-z_{t}^{o}\right)^{2} d z \\
= & \int_{-h / 2+Z_{t}^{o}}^{h / 2+Z_{t}^{o}}[\bar{Q}] z^{2} d z-2 z_{t}^{o} \int_{-h / 2+Z_{t}^{o}}^{h / 2+Z_{t}^{o}}[\bar{Q}] z d z \\
& +\left(z_{t}^{o}\right)^{2}\left[A_{i j}\right]_{t} \quad i, j=1,2,6
\end{aligned}
$$




$$
\left[S_{i j}\right]_{t}=\int_{-h / 2+Z_{t}^{0}}^{h / 2+Z_{t}^{0}}[\bar{Q}] d z, \quad i, j=4,5
$$

where $[\bar{Q}]$ is the transformed reduced stiffness as defined in [22] while $z_{t}^{o}$ is the z-co-ordinate of mid-plane of t-th sublaminate. Thus the formulation based on the multi-point constraint conditions leads to unsymmetric stiffness matrix. The resultant forces and moments at the delamination front for the elements 1,2 and 3 satisfy the following equilibrium conditions [5],

$$
\begin{aligned}
& \{N\}=\{N\}_{1}=\{N\}_{2}+\{N\}_{3} \\
& \{M\}=\{M\}_{1}=\{M\}_{2}+\{M\}_{3}+z_{2}^{\prime}\{N\}_{2}+z_{3}^{\prime}\{N\}_{3} \\
& \{Q\}=\{Q\}_{1}=\{Q\}_{2}+\{Q\}_{3}
\end{aligned}
$$

where $\{Q\}$ denotes the transverse shear resultants. An eight noded isoparametric quadratic plate bending element with five degrees of freedom at each node (three translation and two rotations) is employed wherein the shape functions [10] are given as

$$
\begin{aligned}
& N_{i}=\left(1+\xi \xi_{i}\right)\left(1+\eta \eta_{i}\right)\left(\xi \xi_{i}+\eta \eta_{i}-1\right) / 4(\text { for } i=1,2,3,4) \\
& N_{i}=\left(1-\xi^{2}\right)\left(1+\eta \eta_{i}\right) / 2 \quad(\text { for } i=5,6) \\
& N_{i}=\left(1-\eta^{2}\right)\left(1+\xi \xi_{i}\right) / 2 \quad(\text { for } i=6,8)
\end{aligned}
$$

where $\xi$ and $\eta$ are the local natural coordinates of the element.

\section{Results and discussion}

Non-dimensional natural frequencies for conical shells $(\mathrm{Rx}=\infty)$ having a square plan-form $\left(\mathrm{L} / \mathrm{b}_{o}=1\right)$, curvature ratio $\left(b_{o} / \mathrm{Ry}\right)$ of 0.5 and thickness ratio of $(\mathrm{s} / \mathrm{h})$ of 1000 are obtained corresponding to different speeds of rotation, $\Omega=0.0,0.5$ and 1.0 and relative distance, $\mathrm{d} / \mathrm{L}=0.33,0.5$ and 0.66 , considering three different angles of twist, namely $\psi=15^{\circ}, 30^{\circ}$ and $45^{\circ}$, in addition to the untwisted one $\left(\psi=0^{\circ}\right)$. Parametric studies are carried out with respect to location of delamination, angle of twist and rotational speeds on natural frequencies of angle-ply composite shallow conical shells. The finite element formulation employs eight noded plate bending element with five degrees of freedom at each node. Material properties of graphite-epoxy composite [16] considered as $\mathrm{E}_{1}=$ $138.0 \mathrm{GPa}, \mathrm{E}_{2}=8.96 \mathrm{GPa}, \nu=0.3, \mathrm{G}_{12}=7.1 \mathrm{GPa}, \mathrm{G}_{13}=7.1 \mathrm{GPa}, \mathrm{G}_{23}=2.84 \mathrm{GPa}$. Convergence studies are performed using uniform mesh division of $(6 \times 6)$ and $(8 \times 8)$ and the results are found to be nearly equal, with the difference being around $1 \%$. A comparative study is also conducted to investigate the differences between free vibration characteristics of symmetric and anti-symmetric cross-ply conical shells.

Table 1

Fundamental frequencies $\left[\omega=\omega_{n} \mathrm{~L}^{2} \sqrt{ }\left(\rho / E_{1} h^{2}\right)\right]$ of three layered $[\theta,-\theta$, $\theta]$ graphite- epoxy composite twisted plates, $\mathrm{L} / \mathrm{b}=1, \mathrm{~b} / \mathrm{h}=20, \psi=30^{\circ}$

\begin{tabular}{ccc}
\hline Fibre orientation angle, $\theta$ & Present FEM & Qatu and Leissa [15] \\
\hline $15^{\circ}$ & 0.8618 & 0.8759 \\
$30^{\circ}$ & 0.6790 & 0.6923 \\
$45^{\circ}$ & 0.4732 & 0.4831 \\
$60^{\circ}$ & 0.3234 & 0.3283 \\
\hline
\end{tabular}


Table 2

Convergence study for non-dimensional fundamental frequencies $\left[\omega=\omega_{n} b_{0}^{2} \sqrt{ }(\rho \mathrm{h} / \mathrm{D})\right]$ for graphite-epoxy composite pretwisted shallow conical shell with $\nu=0.3, \mathrm{~s} / \mathrm{h}=1000, \theta_{v}=15^{\circ}$ and $\theta_{o}=30^{\circ}$

\begin{tabular}{ccccc}
\hline$\psi$ & Aspect ratio $(\mathrm{L} / \mathrm{s})$ & Present FEM $(8 \times 8)$ & Present FEM $(6 \times 6)$ & Liew et al. [11] \\
\hline $0^{\circ}$ & 0.6 & 0.3524 & 0.3552 & 0.3599 \\
& 0.7 & 0.2991 & 0.3013 & 0.3060 \\
$30^{\circ}$ & 0.8 & 0.2715 & 0.2741 & 0.2783 \\
& 0.6 & 0.2805 & 0.2834 & 0.2882 \\
& 0.7 & 0.2507 & 0.2528 & 0.2575 \\
& 0.8 & 0.2364 & 0.2389 & 0.2417 \\
\hline
\end{tabular}

Table 3

Non-dimensional fundamental natural frequencies $\left[\omega=\omega_{n} \mathrm{~L}^{2} \sqrt{ }(\rho \mathrm{h} / \mathrm{D})\right]$ of graphite-epoxy composite isotropic rotating cantilever plate, $\mathrm{L} / \mathrm{b}=1, \mathrm{~h} / \mathrm{L}=0.12, \mathrm{D}=\mathrm{Eh}^{3} /\left\{12\left(1-\nu^{2}\right)\right\}, \nu=0.3$

\begin{tabular}{lcc}
\hline Non-dimensional speed $(\Omega)$ & Present FEM & Sreenivasamurthy and Ramamurti [25] \\
\hline 0.0 & 3.4174 & 3.4368 \\
0.2 & 3.4933 & 3.5185 \\
0.4 & 3.7110 & 3.7528 \\
0.6 & 4.0458 & 4.1287 \\
\hline
\end{tabular}

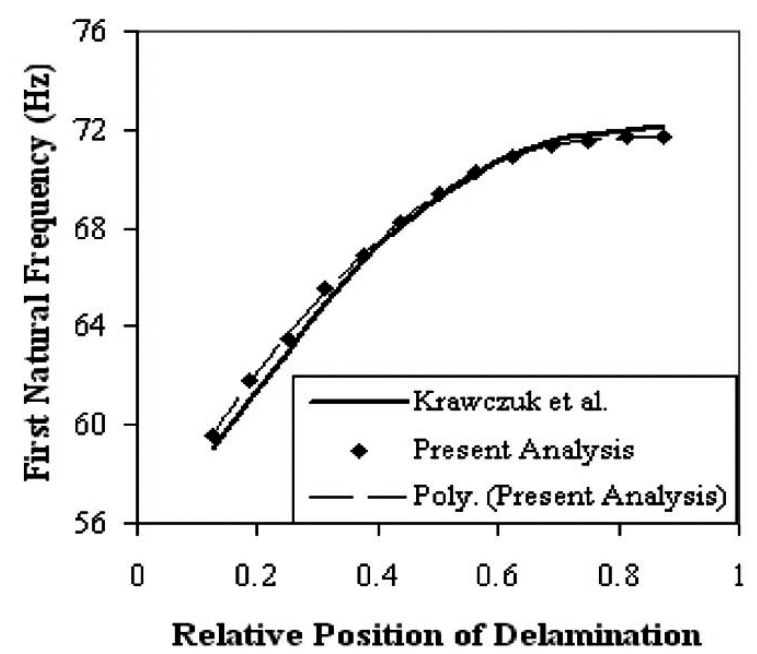

Fig. 3. Influence of the relative position of delamination on the first natural frequency of the composite cantilever beam (Krawczuk et al. [14]).

\subsection{Validation of results}

Based on the present finite element modeling, computer codes are developed and the results obtained are compared and validated with those published in open literature as furnished in Tables 1, 2, 3 and in Fig. 3. The comparative study depicts an excellent agreement with the previously published results and hence it demonstrates the capability of the computer codes in respect of twisted, rotating and delamination models developed for conical shells and ensures the accuracy of analyses. Table 1 furnishes non-dimensional fundamental frequencies of graphite-epoxy composite twisted plates with different fibre-orientation angle [15], while Table 2 presents the convergence study for validation of non-dimensional fundamental natural frequencies of graphite-epoxy composite pretwisted shallow conical shells [11]. On the other hand, Table 3 furnishes the non-dimensional fundamental natural frequencies of isotropic flat rotating cantilever plate [25]. The span-wise variation of fundamental frequency of composite cantilever beam with relative position of delamination [14] is illustrated in Fig. 3. It is observed from the convergence study (Table 2) that uniform mesh divisions of $(6 \times 6)$ and $(8 \times 8)$ considering the complete planform of the shell provide nearly equal results the difference being well within one percent $(1 \%)$. The lower mesh size $(6 \times 6)$ consisting of 36 elements and 133 nodes, has been used for the analysis due to computational efficiency. The total number of degrees 
Table 4

NDFF and NDSF of four layered $\left[0^{\circ} / 90^{\circ} / 0^{\circ} / 90^{\circ}\right]$ graphite-epoxy cross-ply composite pretwisted shallow conical shells by varying relative position of delamination across thickness $(\mathrm{h} / \mathrm{h})$, considering $h=0.0004$, s/h $=1000, \mathrm{~L} / \mathrm{s}=0.7, \mathrm{a} / \mathrm{L}=0.33, \mathrm{~d} / \mathrm{L}=0.5, \nu=0.3, \Omega=0.0, \theta_{o}=45^{\circ}, \theta_{v}=20^{\circ}$

\begin{tabular}{|c|c|c|c|c|c|c|c|c|}
\hline \multirow[t]{2}{*}{$\mathrm{h}^{\prime} / \mathrm{h}$} & \multicolumn{4}{|c|}{ NDFF } & \multicolumn{4}{|c|}{ NDSF } \\
\hline & $\Psi=0^{\circ}$ & $\Psi=15^{\circ}$ & $\Psi=30^{\circ}$ & $\Psi=45^{\circ}$ & $\Psi=0^{\circ}$ & $\Psi=15^{\circ}$ & $\Psi=30^{\circ}$ & $\Psi=45^{\circ}$ \\
\hline 0.00 & 0.35926 & 0.21617 & 0.13063 & 0.08264 & 0.42429 & 0.49170 & 0.35972 & 0.25424 \\
\hline 0.25 & 0.30551 & 0.19346 & 0.11747 & 0.07519 & 0.42389 & 0.42552 & 0.30147 & 0.19759 \\
\hline 0.50 & 0.30767 & 0.19450 & 0.11840 & 0.07613 & 0.42395 & 0.43398 & 0.30998 & 0.20750 \\
\hline 0.75 & 0.30886 & 0.19518 & 0.11903 & 0.07674 & 0.42398 & 0.43908 & 0.31611 & 0.21528 \\
\hline 1.00 & 0.35926 & 0.21617 & 0.13063 & 0.08264 & 0.42429 & 0.49170 & 0.35972 & 0.25424 \\
\hline
\end{tabular}

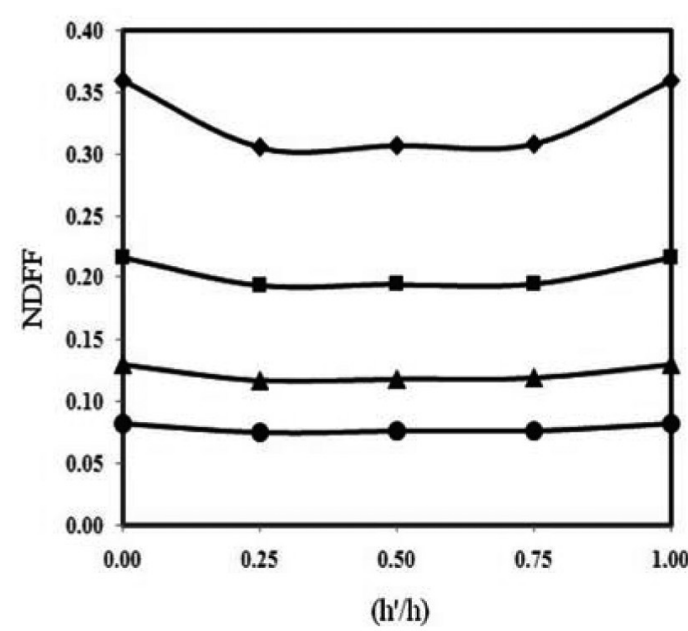

(a) NDFF across thickness for $\mathrm{n}=4$

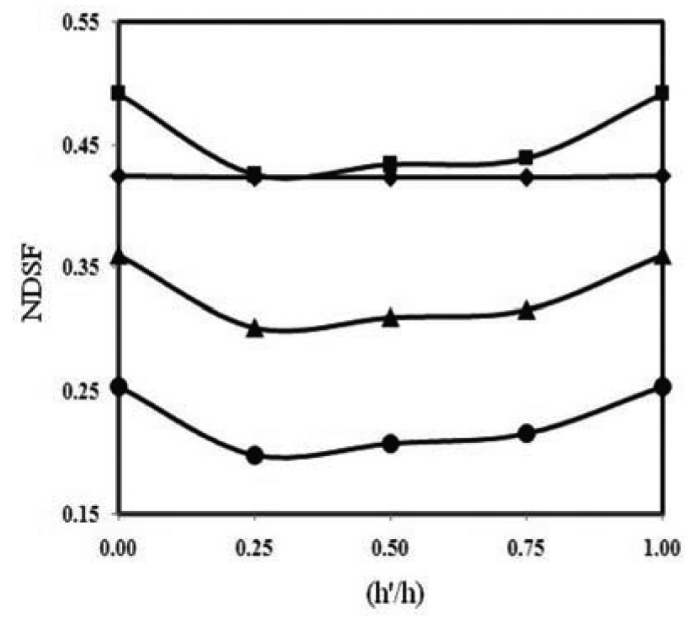

(b) NDSF across thickness for $\mathrm{n}=4$

$$
\rightarrow \psi=0^{\circ} \rightarrow \psi=15^{\circ} \rightarrow \psi=30^{\circ} \rightarrow \psi=45^{\circ}
$$

Fig. 4. (a) NDFF and (b) NDSF of four layered graphite-epoxy cross-ply $\left[0^{\circ} / 90^{\circ} / 0^{\circ} / 90^{\circ}\right]$ composite pretwisted shallow conical shells with respect to relative position of delamination across thickness $\left(\mathrm{h}^{\prime} / \mathrm{h}\right)$, considering $\mathrm{a} / \mathrm{L}=0.33, \mathrm{~d} / \mathrm{L}=0.5, \mathrm{~h}=0.0004, \mathrm{~s} / \mathrm{h}=1000, \mathrm{~L} / \mathrm{s}=0.7, \Omega=$ $0.0, \theta_{o}=45^{\circ}, \theta_{v}=20^{\circ}$.

of freedom involved in the computation is 665 as each node of the isoparametric element is having five degrees of freedom comprising of three translations and two rotations.

\subsection{Location of delamination across thickness}

NDFF and NDSF of graphite-epoxy cross-ply composite stationary conical shells by varying the relative position of the delamination across the thickness for various twist angles are presented in Tables 4, 5 and 6 for $n=4,6$ and 8 , respectively. The results show that the non-dimensional natural frequencies first decreases and then increases as the delamination changes position from the interface near the top surface to the one near the bottom surface of the laminated shell. For any number of layer and at a particular relative position of the delamination across the thickness of the conical shell, it is also observed that the non-dimensional fundamental and second natural frequencies are found to decrease with the increase of twist angle from $0^{\circ}$ to $45^{\circ}$, with an exception for non-dimensional second natural frequencies wherein $\psi=15^{\circ}$ corresponds to maximum value (e.g. Fig. 4 with $n=4$ ).

\subsubsection{Four layered cross-ply conical shells}

From Table 4 and Fig. 4, it is identified that NDFF attains a minimum value at a relative position of 0.25 , i.e. when the delamination is located between the first and second layers for any values of the twist angle. Here, maximum value corresponds to undelaminated case. As the twist angle increases from $\psi=0^{\circ}$ to $\psi=45^{\circ}$, the maximum 
Table 5

NDFF and NDSF of six layered $\left[0^{\circ} / 90^{\circ} / 0^{\circ} / 90^{\circ} / 0^{\circ} / 90^{\circ}\right]$ graphite-epoxy cross-ply composite pretwisted shallow conical shells by varying the relative position of delamination across the thickness $\left(\mathrm{h}^{\prime} / \mathrm{h}\right)$, considering $h=0.0004$, $\mathrm{s} / \mathrm{h}=1000, \mathrm{~L} / \mathrm{s}=0.7, \mathrm{a} / \mathrm{L}=0.33, \mathrm{~d} / \mathrm{L}=0.5, \nu=0.3, \Omega=0.0, \theta_{o}=45^{\circ}, \theta_{v}=20^{\circ}$

\begin{tabular}{|c|c|c|c|c|c|c|c|c|}
\hline \multirow[t]{2}{*}{$\mathrm{h} / \mathrm{h}$} & \multicolumn{4}{|c|}{ NDFF } & \multicolumn{4}{|c|}{ NDSF } \\
\hline & $\Psi=0^{\circ}$ & $\Psi=15^{\circ}$ & $\Psi=30^{\circ}$ & $\Psi=45^{\circ}$ & $\Psi=0^{\circ}$ & $\Psi=15^{\circ}$ & $\Psi=30^{\circ}$ & $\Psi=45^{\circ}$ \\
\hline 0.000 & 0.36010 & 0.21713 & 0.13195 & 0.08416 & 0.42466 & 0.49425 & 0.36315 & 0.25843 \\
\hline 0.166 & 0.32434 & 0.20216 & 0.12331 & 0.07946 & 0.42438 & 0.45164 & 0.32463 & 0.22065 \\
\hline 0.333 & 0.32470 & 0.2024 & 0.12355 & 0.07971 & 0.42441 & 0.45353 & 0.32704 & 0.22380 \\
\hline 0.500 & 0.32503 & 0.20262 & 0.12378 & 0.07993 & 0.42444 & 0.45522 & 0.32929 & 0.22679 \\
\hline 0.666 & 0.32533 & 0.20282 & 0.12399 & 0.08012 & 0.42446 & 0.45677 & 0.33142 & 0.22963 \\
\hline 0.833 & 0.32561 & 0.20302 & 0.12418 & 0.08030 & 0.42448 & 0.45822 & 0.33346 & 0.23236 \\
\hline 1.000 & 0.36010 & 0.21713 & 0.13195 & 0.08416 & 0.42466 & 0.49425 & 0.36315 & 0.25843 \\
\hline
\end{tabular}

Table 6

NDFF and NDSF of eight layered $\left[0^{\circ} / 90^{\circ} / 0^{\circ} / 90^{\circ} / 0^{\circ} / 90^{\circ} / 0^{\circ} / 90^{\circ}\right.$ ] graphite-epoxy cross-ply composite pretwisted shallow conical shells by varying relative position of delamination across thickness $\left(\mathrm{h}^{\prime} / \mathrm{h}\right)$, considering $\mathrm{h}=$ $0.0004, \mathrm{~s} / \mathrm{h}=1000, \mathrm{~L} / \mathrm{s}=0.7, \mathrm{a} / \mathrm{L}=0.33, \mathrm{~d} / \mathrm{L}=0.5, \nu=0.3, \Omega=0.0, \theta_{o}=45^{\circ}, \theta_{v}=20^{\circ}$

\begin{tabular}{|c|c|c|c|c|c|c|c|c|}
\hline \multirow[t]{2}{*}{$\mathrm{h} / \mathrm{h}$} & \multicolumn{4}{|c|}{ NDFF } & \multicolumn{4}{|c|}{ NDSF } \\
\hline & $\Psi=0^{\circ}$ & $\Psi=15^{\circ}$ & $\Psi=30^{\circ}$ & $\Psi=45^{\circ}$ & $\Psi=0^{\circ}$ & $\Psi=15^{\circ}$ & $\Psi=30^{\circ}$ & $\Psi=45^{\circ}$ \\
\hline 0.000 & 0.36386 & 0.22139 & 0.13732 & 0.09014 & 0.42609 & 0.50539 & 0.37722 & 0.27541 \\
\hline 0.125 & 0.33696 & 0.21026 & 0.13105 & 0.08692 & 0.42586 & 0.47476 & 0.34944 & 0.24875 \\
\hline 0.250 & 0.33696 & 0.21026 & 0.13105 & 0.08692 & 0.42586 & 0.47476 & 0.34944 & 0.24875 \\
\hline 0.375 & 0.33696 & 0.21026 & 0.13105 & 0.08692 & 0.42586 & 0.47476 & 0.34944 & 0.24875 \\
\hline 0.500 & 0.33696 & 0.21026 & 0.13105 & 0.08692 & 0.42586 & 0.47476 & 0.34944 & 0.24875 \\
\hline 0.625 & 0.33696 & 0.21026 & 0.13105 & 0.08692 & 0.42586 & 0.47476 & 0.34944 & 0.24875 \\
\hline 0.750 & 0.33696 & 0.21026 & 0.13105 & 0.08692 & 0.42586 & 0.47476 & 0.34944 & 0.24875 \\
\hline 0.875 & 0.33696 & 0.21026 & 0.13105 & 0.08692 & 0.42586 & 0.47476 & 0.34944 & 0.24875 \\
\hline 1.000 & 0.36386 & 0.22139 & 0.13732 & 0.09014 & 0.42609 & 0.50539 & 0.37722 & 0.27541 \\
\hline
\end{tabular}

percentage differences between non-dimensional fundamental frequencies for a particular value of relative position of delamination across the thickness were found $75.4 \%, 75.3 \%$ and $75.2 \%$ corresponding to $\mathrm{h}^{\prime} / \mathrm{h}=0.25,0.5$ and 0.75 while the same is identified as $77.0 \%$ corresponding to $h^{\prime} / h=0.0$ and 1.0 . Similarly, as the twist angle increases from $\psi=0^{\circ}$ to $\psi=45^{\circ}$, the maximum percentage differences between non-dimensional second natural frequencies for a particular value of relative position of delamination across the thickness were found $53.6 \%, 52.2 \%$ and $51.0 \%$ corresponding to $\mathrm{h}^{\prime} / \mathrm{h}=0.25,0.5$ and 0.75 while the same is found as $48.3 \%$ corresponding to $\mathrm{h}^{\prime} / \mathrm{h}=0.0$ and 1.0 . The percentage difference between maximum and minimum values of non-dimensional fundamental frequencies are found $15.0 \%, 10.5 \%, 10.1 \%$ and $9.0 \%$ corresponding to $\psi=0^{\circ}, 15^{\circ}, 30^{\circ}$ and $45^{\circ}$, respectively. On the other hand, percentage difference between maximum and minimum values of non-dimensional second natural frequencies are found $0.1 \%, 13.5 \%, 16.2 \%$ and $22.3 \%$ corresponding to $\psi=0^{\circ}, 15^{\circ}, 30^{\circ}$ and $45^{\circ}$, respectively. It is observed that as the twist angle increases, the percentage difference between maximum and minimum values of non-dimensional fundamental frequencies decreases. In other words, the maximum effect of delamination is manifested in untwisted shell when the location of delamination changes across the thickness. In contrast, the percentage difference between maximum and minimum values of non-dimensional second natural frequencies increase as the twist angles also increase. So the maximum effect of delamination in respect of NDSF is found in case of twisted shell of $\psi=45^{\circ}$.

\subsubsection{Six layered cross-ply conical shells}

From Table 5, it is identified that NDFF attains a minimum value at a relative position of $\mathrm{h}^{\prime} / \mathrm{h}=0.166$, i.e. when the delamination is located between the first and second layers for any values of the twist angle. In this case, maximum value is also observed at two free surfaces (i.e., undelaminated cases). As the twist angle increases from $\psi=0^{\circ}$ to $\psi=45^{\circ}$, the maximum percentage differences between non-dimensional fundamental frequencies for a particular value of relative position of delamination across the thickness were found 75.5\%, 75.5\%, 75.4\%, $75.4 \%$ and $75.3 \%$ corresponding to $\mathrm{h}^{\prime} / \mathrm{h}=0.166,0.333,0.5,0.666$ and 0.833 while the same is found as $76.6 \%$ corresponding to $\mathrm{h}^{\prime} / \mathrm{h}=0.0$ and 1.0. Similarly, as the twist angle increases from $\psi=0^{\circ}$ to $\psi=45^{\circ}$, the maximum percentage differences between non-dimensional second natural frequencies for a particular value of relative position 
Table 7

NDFF and NDSF of symmetric eight layered $\left[0^{\circ} / 90^{\circ} / 0^{\circ} / 90^{\circ} / 90^{\circ} / 0^{\circ} / 90^{\circ} / 0^{\circ}\right]$ graphite-epoxy cross-ply composite pretwisted shallow conical shells, with $\mathrm{a} / \mathrm{L}=0.33$ and $\mathrm{d} / \mathrm{L}=0.33,0.66$ from the fixed end, by varying the relative position of delamination along the span, considering $\mathrm{h}=0.0004, \mathrm{~s} / \mathrm{h}=1000, \mathrm{~L} / \mathrm{s}=0.7, \theta_{o}=45^{\circ}, \theta_{v}=20^{\circ}$

\begin{tabular}{|c|c|c|c|c|c|}
\hline \multirow[t]{2}{*}{$\Psi$} & \multirow[t]{2}{*}{$\Omega$} & \multicolumn{2}{|c|}{ NDFF } & \multicolumn{2}{|c|}{ NDSF } \\
\hline & & $\mathrm{d} / \mathrm{L}=0.33$ & $\mathrm{~d} / \mathrm{L}=0.66$ & $\mathrm{~d} / \mathrm{L}=0.33$ & $\mathrm{~d} / \mathrm{L}=0.66$ \\
\hline \multirow[t]{3}{*}{$0^{\circ}$} & 0.0 & 0.33523 & 0.34922 & 0.42543 & 0.42584 \\
\hline & 0.5 & 0.42151 & 0.42157 & 0.69746 & 0.67385 \\
\hline & 1.0 & 0.27605 & 0.38876 & 0.39361 & 0.81670 \\
\hline \multirow[t]{3}{*}{$15^{\circ}$} & 0.0 & 0.20705 & 0.21561 & 0.49229 & 0.46436 \\
\hline & 0.5 & 0.31722 & 0.43126 & 0.70715 & 0.56448 \\
\hline & 1.0 & 0.45441 & 0.43969 & 0.61729 & 0.58614 \\
\hline \multirow[t]{3}{*}{$30^{\circ}$} & 0.0 & 0.12751 & 0.13338 & 0.36423 & 0.34535 \\
\hline & 0.5 & 0.32032 & 0.28541 & 0.57026 & 0.55626 \\
\hline & 1.0 & 0.29378 & 0.27482 & 0.45890 & 0.57181 \\
\hline \multirow[t]{3}{*}{$45^{\circ}$} & 0.0 & 0.08353 & 0.08736 & 0.26145 & 0.24992 \\
\hline & 0.5 & 0.23550 & 0.18717 & 0.45500 & 0.43183 \\
\hline & 1.0 & 0.21402 & 0.17574 & 0.45920 & 0.43471 \\
\hline
\end{tabular}

of delamination across the thickness were found $51.1 \%, 50.7 \%, 50.2 \%, 49.7 \%$ and $49.3 \%$ corresponding to $\mathrm{h}^{\prime} / \mathrm{h}=$ $0.166,0.333,0.5,0.666$ and 0.833 while the same is obtained as $47.7 \%$ corresponding to $\mathrm{h}^{\prime} / \mathrm{h}=0.0$ and 1.0 . So, it can be inferred that this parameter is invariant with twist angle. On the other hand, the percentage difference between maximum and minimum values of non-dimensional fundamental frequencies are found $9.9 \%, 6.9 \%, 6.5 \%$ and $5.6 \%$ corresponding to $\psi=0^{\circ}, 15^{\circ}, 30^{\circ}$ and $45^{\circ}$, respectively. On the other hand, percentage difference between maximum and minimum values of non-dimensional second natural frequencies are found $0.1 \%, 8.6 \%, 10.6 \%$ and $14.6 \%$ corresponding to $\psi=0^{\circ}, 15^{\circ}, 30^{\circ}$ and $45^{\circ}$, respectively. So, it can be said that even with more number of layers, i.e., $n=6$, delamination effect is more pronounced in case of untwisted shell corresponding to fundamental frequency as observed for $n=4$.

\subsubsection{Eight layered cross-ply conical shells}

In this case, it is also observed that NDFF and NDSF have a constant value for a particular value of twist angle when the location of delamination changes across the thickness. In other words, maximum value is obtained at two free surfaces and the minimum value which is constant in nature is found throughout the thickness. Like the case of four and six layers, for eight layered laminates, non-dimensional fundamental frequency decreases with the increase of twist angle, whereas the non-dimensional second natural frequency attains maximum value at $\Psi=15^{\circ}$ and decreases to a minimum at $\Psi=45^{\circ}$. It is to be noted remarkably that the delaminated frequencies (both fundamental and second) remain invariant with the change of location of delamination across the thickness corresponding to twisted and untwisted shells. This might be attributed to the fact that the sub-laminates of the unsymmetric cross-ply composite shells analyzed always contribute in the same proportions in respect of structural stiffness and thereby lead to the degradation of stiffness due to delamination by equal amount.

\subsection{Location of delamination along span}

A delamination of relative length $\mathrm{a} / \mathrm{L}=0.33$ is considered and this is centered at a relative distances of $\mathrm{d} / \mathrm{L}=$ 0.33 and 0.66 from the fixed end. The delamination is considered at the mid-plane of symmetric eight layered crossply of graphite-epoxy composite pre-twisted rotating conical shells (Table 7). At stationary condition, NDFF are observed to decrease with the increase of twist angle. At stationary condition, non-dimensional fundamental natural frequencies for both twisted and untwisted conical shell are found to increase as the delamination moves towards the free end while the reverse is noted for second natural frequency except for $\Psi=0^{\circ}$. In case of fundamental natural frequency, the centrifugal stiffening effect is observed for $\Psi=15^{\circ}$ corresponding to $\mathrm{d} / \mathrm{L}=0.33$ and 0.66 but in case of second natural frequency, the centrifugal stiffening effect is found for $\Psi=0^{\circ}, 15^{\circ}$ and $30^{\circ}$ corresponding to $\mathrm{d} / \mathrm{L}=0.66$ and for $\Psi=45^{\circ}$ corresponding to $\mathrm{d} / \mathrm{L}=0.33$ and 0.66 . At lower rotational speed $(\Omega=0.5)$, non-dimensional fundamental natural frequencies are observed to increase as the delamination moves towards the free end corresponding to $\Psi=0^{\circ}, 15^{\circ}$ except for $\Psi=30^{\circ}, 45^{\circ}$, while at higher rotational speed $(\Omega=1.0)$, 
Table 8

Comparative statement for NDFF of four layered a) Symmetric and b) Anti-symmetric graphite-epoxy cross-ply composite pretwisted shallow conical shells, considering $h=0.0004, \mathrm{~s} / \mathrm{h}=1000, \mathrm{~L} / \mathrm{s}=0.7, \mathrm{~d} / \mathrm{L}=0.5, \nu=0.3$, $\theta_{o}=45^{\circ}, \theta_{v}=20^{\circ}$

\begin{tabular}{|c|c|c|c|c|c|c|c|c|}
\hline \multirow[t]{3}{*}{$\Psi$} & \multicolumn{4}{|c|}{ Symmetric $\left(0^{\circ} / 90^{\circ} / 90^{\circ} / 0^{\circ}\right)$} & \multicolumn{4}{|c|}{ Anti-symmetric $\left(0^{\circ} / 90^{\circ} / 0^{\circ} / 90^{\circ}\right)$} \\
\hline & \multirow[t]{2}{*}{ ND } & \multicolumn{3}{|c|}{ Mid-plane Delamination } & \multirow[t]{2}{*}{ ND } & \multicolumn{3}{|c|}{ Mid-plane Delamination } \\
\hline & & $\Omega=0.0$ & $\Omega=0.5$ & $\Omega=1.0$ & & $\Omega=0.0$ & $\Omega=0.5$ & $\Omega=1.0$ \\
\hline $0^{\circ}$ & 0.4270 & 0.3154 & 0.4233 & 0.3210 & 0.4242 & 0.3076 & 0.4214 & 0.3109 \\
\hline $15^{\circ}$ & 0.2245 & 0.2029 & 0.3064 & 0.4144 & 0.2161 & 0.1945 & 0.3592 & 0.4554 \\
\hline $30^{\circ}$ & 0.1409 & 0.1291 & 0.2740 & 0.2930 & 0.1306 & 0.1184 & 0.2748 & 0.2272 \\
\hline $45^{\circ}$ & 0.0941 & 0.0881 & 0.2066 & 0.2016 & 0.0826 & 0.0761 & 0.1469 & 0.0333 \\
\hline
\end{tabular}

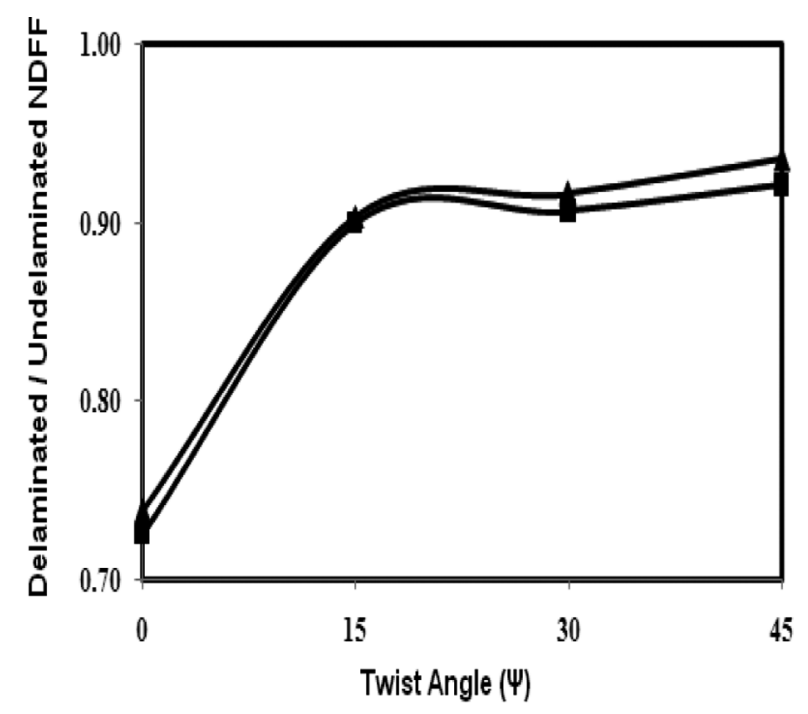

\section{$\mp$ Symmetric $\rightarrow$ Anti-symmetric}

Fig. 5. Comparative representation of ratio of delaminated and undelaminated NDFF at stationary condition for symmetric and anti-symmetric four layered graphite-epoxy cross-ply composite pretwisted shallow conical shells, considering $\mathrm{h}=0.0004, \mathrm{~s} / \mathrm{h}=1000, \mathrm{~L} / \mathrm{s}=0.7, \mathrm{~d} / \mathrm{L}=0.5$, $\theta_{o}=45^{\circ}, \theta_{v}=20^{\circ}$.

non-dimensional fundamental frequencies are found to decrease as the delamination moves towards the free end corresponding to $\Psi=15^{\circ}, 30^{\circ}, 45^{\circ}$ except for $\Psi=0^{\circ}$. On the other hand, for lower and higher rotational speed, non-dimensional second natural frequencies are observed to decrease as the delamination moves towards the free end irrespective of twist angle. The centrifugal stiffening effect is observed for NDFF of symmetric cross-ply laminate corresponding to $\Psi=15^{\circ}$ for both $\mathrm{d} / \mathrm{L}=0.33$ and 0.67 , while for NDSF symmetric case, the same is identified throughout at $\mathrm{d} / \mathrm{L}=0.67$ for $\Psi=0^{\circ}, 15^{\circ}, 30^{\circ}$ and $45^{\circ}$ and at $\mathrm{d} / \mathrm{L}=0.33$ for $\Psi=45^{\circ}$.

\subsection{Effect of rotational speed and twist angle}

In general, for a stationary cross-ply composite shallow conical shell, non-dimensional fundamental natural frequency parameters are observed to decrease with the increase of twist angle. A comparative study is conducted to identify the differences in non-dimensional natural frequencies between symmetric and anti-symmetric four layered cross-ply laminates considering both twisted and untwisted cases, as depicted in Table 8. Some other aspects are furnished in Figs 5 and 6. At stationary conditions for both symmetric and anti-symmetric laminates, undelaminated and delaminated non-dimensional natural frequencies are found to achieve maximum values for $\psi=0^{\circ}$ and gradually decrease to a minimum values for $\psi=45^{\circ}$.

The centrifugal stiffening effect is observed for symmetric cross-ply corresponding to $\Psi=15^{\circ}$ and $30^{\circ}$ while for anti-symmetric cases, the same is identified for $\Psi=15^{\circ}$ only. On the other hand at stationary condition, 


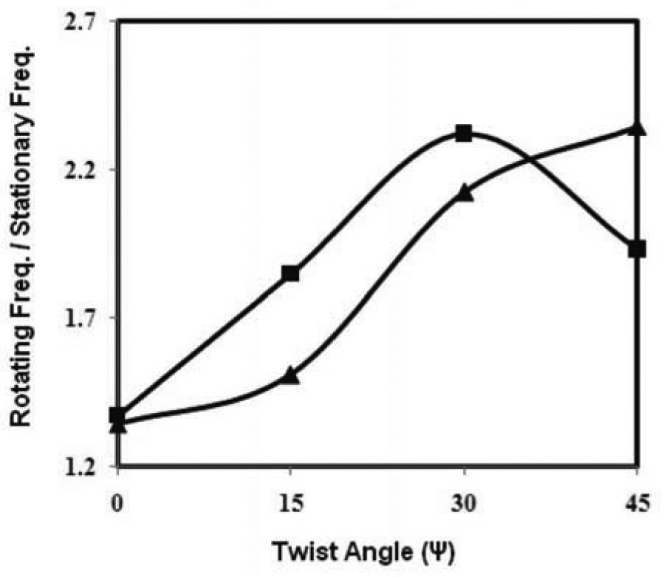

(a) At Lower Rotational speed $(\Omega=0.5)$

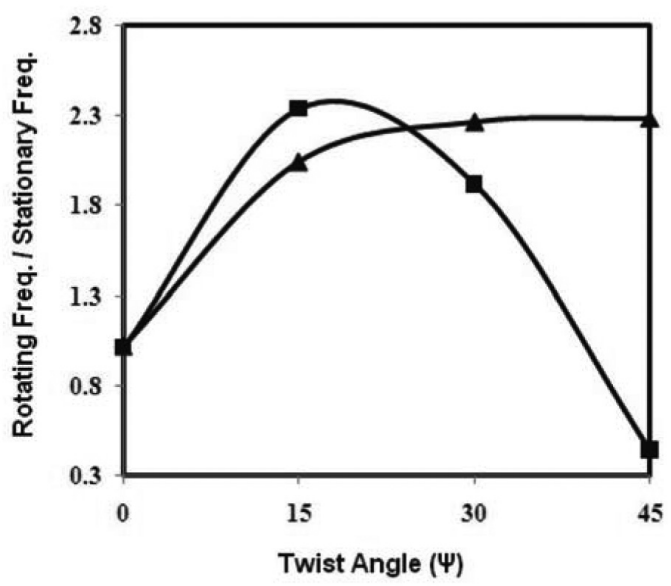

(b) At Higher Rotational Speed $(\Omega=1.0)$

\section{$\rightarrow$ Symmetric}

\section{$\rightarrow-$ Anti-symmetric}

Fig. 6. Comparative representation of ratio of rotating frequency and stationary frequency for symmetric and anti-symmetric four layered graphite-epoxy cross-ply composite pretwisted rotating shallow conical shells, considering $\mathrm{h}=0.0004, \mathrm{~s} / \mathrm{h}=1000, \mathrm{~L} / \mathrm{s}=0.7, \mathrm{~d} / \mathrm{L}=0.5, \theta_{o}=$ $45^{\circ}, \theta_{v}=20^{\circ}$.

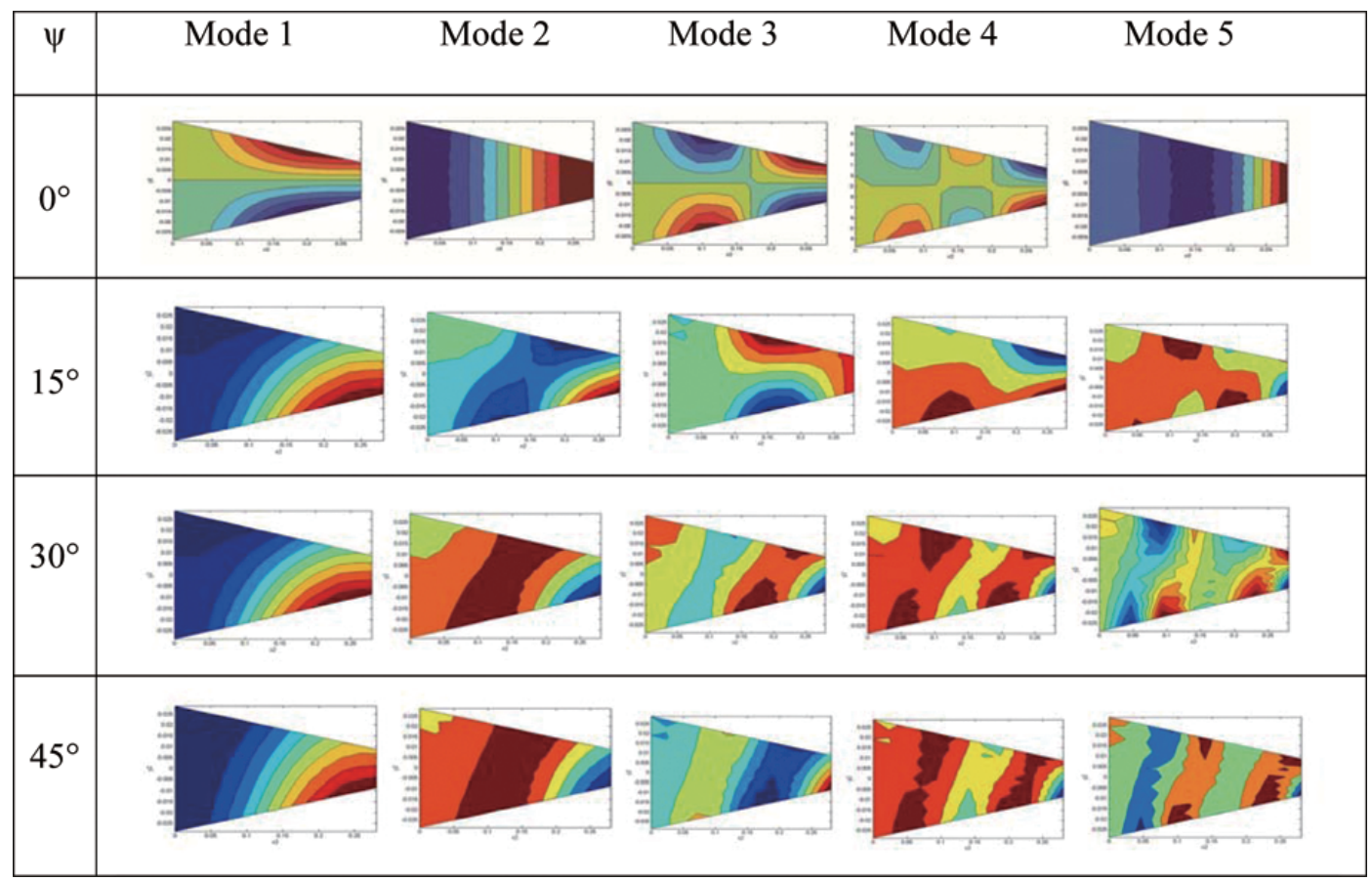

Fig. 7. Effects of twist angles on mode shapes for NDFF of symmetric six layered $\left[0^{\circ} / 90^{\circ} / 0^{\circ} / 0^{\circ} / 90^{\circ} / 0^{\circ}\right]$ graphite-epoxy cross-ply composite shallow conical shells with mid-plane delamination at stationary condition, considering $\mathrm{h}=0.0004, \mathrm{~s} / \mathrm{h}=1000, \mathrm{~L} / \mathrm{s}=0.7, \mathrm{~d} / \mathrm{L}=0.5, \nu=0.3$, $\theta_{o}=45^{\circ}, \theta_{v}=20^{\circ}$.

non-dimensional fundamental frequencies of symmetric cross-ply laminates are identified to be higher than nondimensional fundamental frequencies of anti-symmetric cross-ply laminates. The percentage difference between maximum and minimum relative frequencies at stationary condition is found $21.2 \%$ for both symmetric and antisymmetric cross-ply laminates. 
At lower rotational speed $(\Omega=0.5)$, the percentage difference between maximum and minimum value of rotating frequencies and stationary frequencies are worked out to $42.8 \%$ and $41.0 \%$, while for higher rotational speed $(\Omega=$ 1.0), the same values found $55.5 \%$ and $81.3 \%$ for symmetric and anti-symmetric cases respectively irrespective of twist angle. Hence it leads to the fact that for higher rotational speed, stacking sequence has got pronounced effect in respect of the non-dimensional natural frequencies. It is also to be noted that delaminated non-dimensional natural frequencies found lower than undelaminated natural frequencies at stationary condition for both symmetric and anti-symmetric cases. At stationary condition, the percentage difference between undelaminated non-dimensional fundamental frequencies found $78 \%$ and $80.5 \%$ corresponds to symmetric and anti-symmetric cases respectively, while the same for delaminated non-dimensional fundamental frequencies found $72.1 \%$ and $75.3 \%$ corresponds to symmetric and anti-symmetric cases respectively. Hence it is evident that for undelaminated cases, twisted effect has got more pronounced effect than delaminated cases in respect of the non-dimensional natural frequencies.

\subsection{Mode shapes}

The different modes are plotted in Fig. 7 considering six layered symmetric cross-ply composite pretwisted shallow conical shell with mid-plane delamination at stationary condition. In this case, the fundamental mode corresponds to the first torsion. It is identified that the symmetry modes are absent when twist angle is non-zero, but present for untwisted case and the nodal lines indicate with zero displacement amplitude. The first span wise bending is observed only for untwisted conical shell corresponding to its second and fifth natural frequencies. The dominance of torsional mode is found significantly for all twisted cases corresponding to fundamental natural frequencies.

\section{Conclusions}

The present study on delaminated cross ply composite twisted conical shells without considering the effect of the dynamic contact of the delaminated layers leads to the following conclusions:

1. The close agreement of the results obtained by the present method with those available in the published literature establishes the correctness of the approach used here.

2. The non-dimensional natural frequency initially decreases to a minimum and then increases as the delamination changes position from the interface near the top surface to the one near the bottom surface of the laminated shell. For any number of layers and at a particular relative position of the delamination across the thickness of the conical shell, the non-dimensional fundamental and second natural frequencies are found to decrease with the increase of twist angle. The maximum effect of delamination is manifested in untwisted shell when the location of delamination changes across the thickness.

3. It is to be noted remarkably that for eight layered conical shells the delaminated frequencies (both fundamental and second) remain invariant with the change of location of delamination across the thickness corresponding to both twisted and untwisted cases.

4. At stationary condition, non-dimensional fundamental natural frequencies are observed to decrease with the increase of twist angle.

5. At stationary condition, non-dimensional fundamental natural frequencies for both twisted and untwisted conical shell are found to increase as the delamination moves along the span from the fixed end to the free end while the reverse is noted for second natural frequency except for untwisted cases.

6. As expected the delaminated non-dimensional natural frequencies are found lower than the undelaminated non-dimensional natural frequencies at stationary condition for both symmetric and anti-symmetric cases. At higher rotational speed, non-dimensional fundamental frequencies are found to decrease for the twisted shells as the delamination moves along the span towards the free end, while in contrast non-dimensional second natural frequencies are observed to decrease irrespective of twist angle.

7. From the mode shapes of six layered symmetric pretwisted cross-ply shallow conical shell with mid-plane delamination at stationary condition, it is observed that the fundamental mode corresponds to the first torsion and the dominance of torsional mode is found significantly for all twisted cases corresponding to fundamental natural frequencies. 


\section{References}

[1] A. Karmakar and K. Kishimoto, Free Vibration Analysis of Delaminated Composite, JSME International Journal, Series A 49(4) (2006), 492-502.

[2] A. Karmakar and P.K. Sinha, Failure Analysis of Laminated Composite Pretwisted Rotating Plates, Journal of Reinforced Plastics and Composites 20 (2001), 1326-1357.

[3] A. Todoroki, Y. Tanaka and Y. Shimamura, Delamination monitoring of graphite/epoxy laminated composite plate of electric resistance change method, Journal of Composites Science and Technology 62(Issue 9) (2002), 1151-1160.

[4] A.W. Leissa, J.K. Lee and A.J. Wang, Vibrations of Twisted Rotating Blades, Journal of Vibration, Acoustics, Stress, and Reliability in Design, Trans., ASME 106(2) (1984), 251-257.

[5] C.K. Gim, Plate Finite Element Modelling of Laminated Plates, Journal of Computers and Structures 52(1) (1994), 157-168.

[6] F. Aymerich, F. Dore and P. Priolo, Simulation of multiple delamination in impacted cross-ply laminates using a finite element model based on cohesive interface elements, Journal of Composites Science and Technology 69 (2009), 1699-1709.

[7] J.J. Lee, C.H. Yeom and I. Lee, Vibration analysis of twisted cantilever conical composite shells, Journal of Sound and Vibration 255 (2002), 965-982.

[8] J.L. Rebière and D. Gamby, A criterion for modelling initiation and propagation of matrix cracking and delamination in cross-ply laminates, Journal of Composites Science and Technology 64(Issues 13-14) (2004), 2239-2250.

[9] J.M. Berthelot and J.F. Le Corre, Statistical analysis of the progression of transverse cracking and delamination in cross-ply laminates, Journal of Composites Science and Technology 60(Issue 14) (2000), 2659-2669.

[10] K.J. Bathe, Finite Element Procedures in Engineering Analysis, PHI, New Delhi, 1990.

[11] K.M. Liew, C.M. Lim and L.S. Ong, Vibration of pretwisted cantilever shallow conical shells, International Journal of Solids Structures 31 (1994), 2463-2474.

[12] M. Aydogdu and T. Timarci, Vibration analysis of cross-ply laminated square plates with general boundary conditions, Journal of Composites Science and Technology 63(Issue 7) (2003), 1061-1070.

[13] M.H.H. Shen and J.E. Grady, Free Vibrations of Delaminated Beams, AIAA Journal 30(5) (1992), 1361-1370.

[14] M. Krawczuk, W. Ostachowicz and A. Zak, Dynamics of Cracked Composite Material Structures, Journal of Computational Mechanics 20 (1997), 79-83.

[15] M.S. Qatu and A.W. Leissa, Vibration studies for Laminated Composite Twisted Cantilever Plates, International Journal of Mechanical Sciences 33 (1991), 927-940.

[16] M.S. Qatu and A.W. Leissa, Natural Frequencies for Cantilevered Doubly-Curved Laminated Composite Shallow Shells, Composite Structures 17 (1991), 227-255.

[17] N. Takeda and S. Ogihara, Initiation and growth of delamination from the tips of transverse cracks in CFRP cross-ply laminates, Composites Science and Technology 52 (1994), 309-318.

[18] N. Toyama, J. Noda and T. Okabe, Quantitative damage detection in cross-ply laminates using Lamb wave method, Composites Science and Technology 63 (2003), 1473-1479.

[19] O.G. McGee and H.R. Chu, Three-Dimensional Vibration Analysis of Rotating Laminated Composite Blades, Journal of Engineering for Gas Turbines and Power, Trans ASME 116 (1994), 663-671.

[20] P.K. Parhi, S.K. Bhattacharyya and P.K. Sinha, Failure analysis of multiple delaminated due to bending and impact, Bull Mater Sci, Indian Academy of Sciences 24 (2001), 143-149.

[21] R.D. Cook, D.S. Malkus and M.E. Plesha, Concepts and Applications of Finite Element Analysis, John Wiley and Sons, New York, 1989.

[22] R.M. Jones, “Mechanics of Composite Materials", Hemisphere, New York, 1975, pp. 147-156.

[23] S. Liang, H.L. Chen, T. Chen and M.Y. Wang, The natural vibration of a symmetric cross-ply laminated composite conical-plate shell, Journal of Composite structures $\mathbf{8 0}$ (2006), 265-278.

[24] S. Sallam and G.J. Simitses, Delamination buckling and growth of flat, cross-ply laminates, Journal of Composite Structures 4(Issue 4) (1985), 361-381.

[25] S. Sreenivasamurthy and V. Ramamurti, Coriolis Effect on the Vibration of Flat Rotating Low Aspect Ratio Cantilever Plates, Journal of Strain Analysis 16 (1981), 97-106.

[26] V. Tripathi, B.N. Singh and K.K. Shukla, Free vibration of laminated composite conical shells with random material properties, Journal of Composite Structures 81 (2007), 96-104.

[27] Y. Narita, Y. Ohta and M. Saito, Finite element study for natural frequencies of cross-ply laminated cylindrical shells, Journal of Composite Structures 26 (Issues 1-2) (1993), 55-62. 

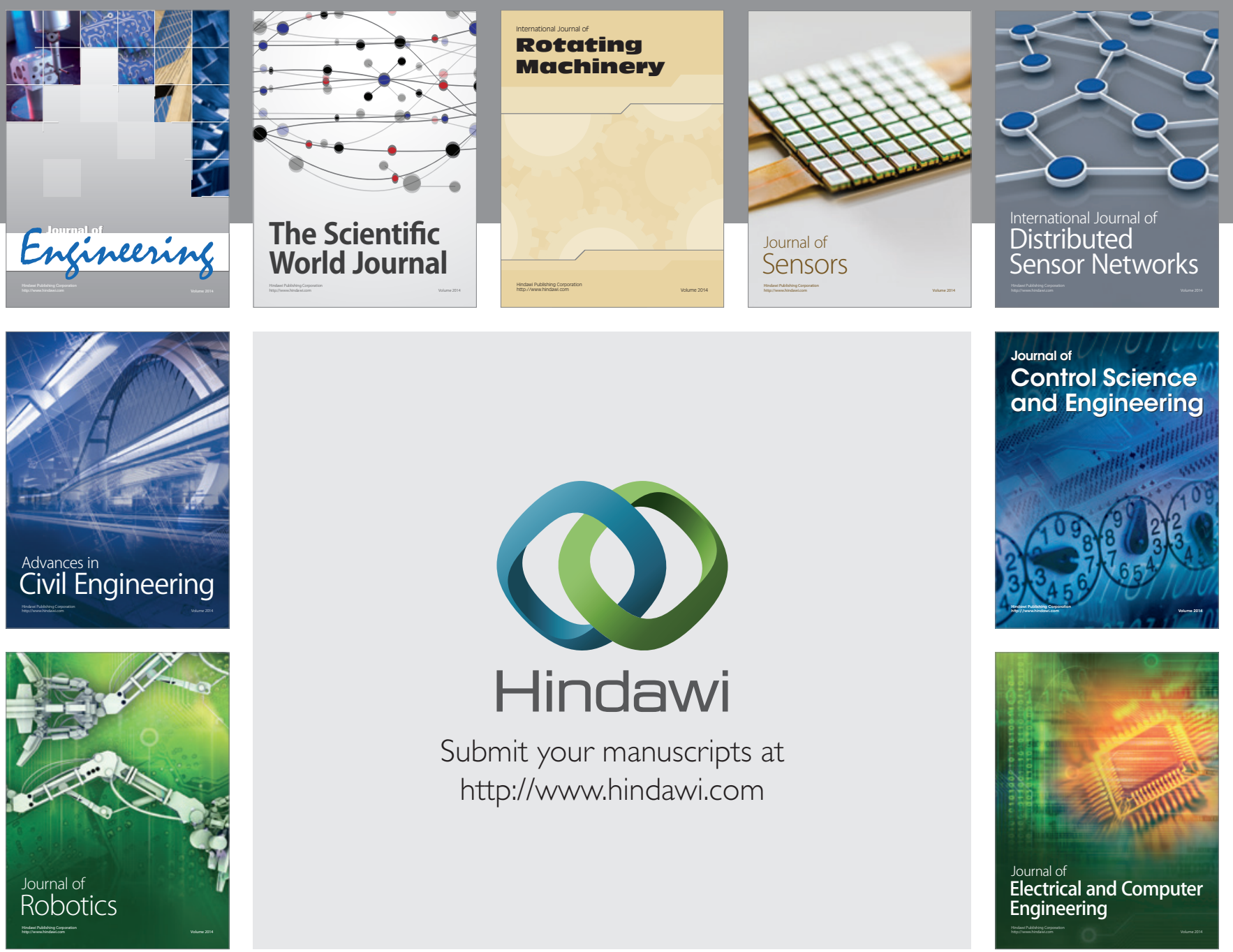

Submit your manuscripts at

http://www.hindawi.com
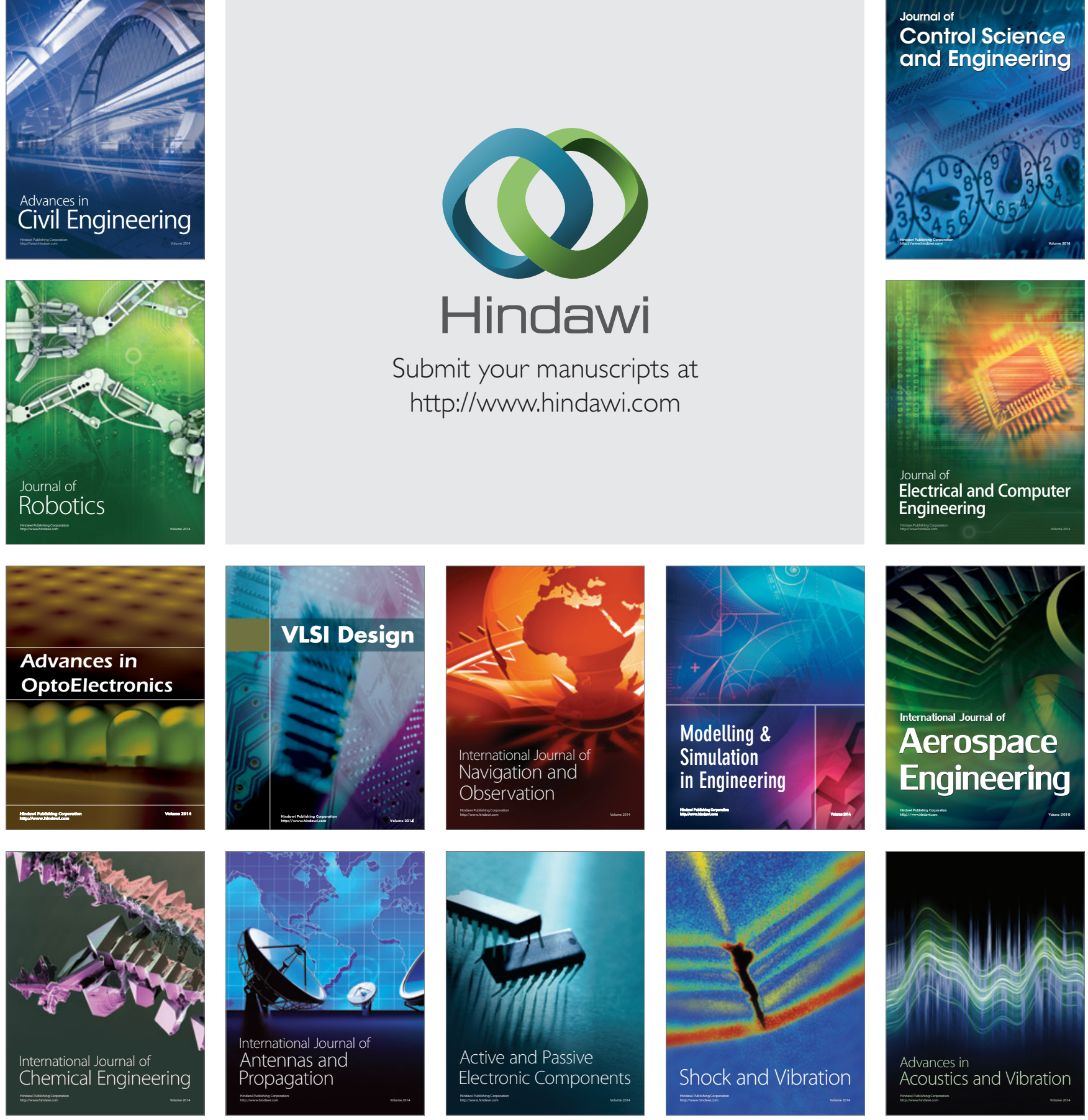